\title{
Three-Dimensional Microfabrication of Protein Hydrogels via Two-Photon-Excited Thiol-Vinyl Ester Photopolymerization
}

\author{
Xiao-Hua Qin, ${ }^{1,2}$ Jan Torgersen, ${ }^{2,3}$ Robert Saf, ${ }^{4}$ Severin Mühleder, ${ }^{2,5}$ Niklas Pucher, ${ }^{1}$ \\ S. Clark Ligon, ${ }^{1,2}$ Wolfgang Holnthoner, ${ }^{2,5}$ Heinz Redl, ${ }^{2,5}$ Aleksandr Ovsianikov, ${ }^{2,3}$ \\ Jürgen Stampfl, ${ }^{2,3}$ Robert Liska ${ }^{1,2}$

\begin{abstract}
${ }^{1}$ Institute of Applied Synthetic Chemistry, Vienna University of Technology, Getreidemarkt 9/163/MC, 1060 Vienna, Austria
${ }^{2}$ Austrian Cluster for Tissue Regeneration

${ }^{3}$ Institute of Materials Science and Technology, Vienna University of Technology, Favoritenstraße 9, 1040 Vienna, Austria

${ }^{4}$ Institute for Chemistry and Technology of Materials, Graz University of Technology, Stremayrgasse 9, 8010 Graz, Austria

${ }^{5}$ Ludwig Boltzmann Institute for Experimental and Clinical Traumatology, Donaueschingenstraße 13, 1200 Vienna, Austria

Correspondence to: R. Liska (E-mail: robert.liska@tuwien.ac.at)
\end{abstract}

Received 17 April 2013; accepted 5 August 2013; published online 2 September 2013

DOI: 10.1002/pola.26903

\begin{abstract}
Engineering three-dimensional (3D) hydrogels with well-defined architectures has become increasingly important for tissue engineering and basic research in biomaterials science. To fabricate 3D hydrogels with (sub)cellular-scale features, two-photon polymerization (2PP) shows great promise although the technique is limited by the selection of appropriate hydrogel precursors. In this study, we report the synthesis of gelatin hydrolysate vinyl esters (GH-VE) and its copolymerization with reduced derivatives of bovine serum albumin (acting as macrothiols). Photorheology of the thiol-ene copolymerization shows a much more rapid onset of polymerization and a higher end modulus in reference to neat GH-VE. This allowed 2PP to provide well-defined and stable hydrogel microstructures. Efficiency of the radical-mediated thiol-vinyl
\end{abstract}

ester photopolymerization allows high 2PP writing speed (as high as $50 \mathrm{~mm} \mathrm{~s}^{-1}$ ) with low laser power (as low as $20 \mathrm{~mW}$ ). MTT assays indicate negligible cytotoxicities of the GH-VE macromers and of the thiol-ene hydrogel pellets. Osteosarcoma cells seeded onto GH-VE/BSA hydrogels with different macromer relative ratios showed a preference for hydrogels with higher percentage of GH-VE. This can be attributed both to a favorable modulus and preferable protein environment since gelatin favors cell adhesion and albumin incurs nonspecific binding. (c) 2013 Wiley Periodicals, Inc. J. Polym. Sci., Part A: Polym. Chem. 2013, 51, 4799-4810

KEYWORDS: biomaterials; gelatin; hydrogels; photopolymerization; tissue engineering; two-photon polymerization; vinyl ester
INTRODUCTION The development of three-dimensional (3D) biomaterial scaffolds capable of promoting cell viability and mimicking certain cell-extracellular matrix (ECM) interactions is of great importance for tissue engineering applications. $^{1,2}$ In particular, there are wide interests in designing 3D hydrogel scaffolds with both structural and biological control to mimic the natural ECM's functions for soft tissue regeneration and 3D cell culture.,

From an engineering standpoint, the lack of a general approach to rapidly construct 3D engineered hydrogel scaffolds remains a major challenge for tissue regeneration. ${ }^{5-7}$ Among the available techniques for 3D fabrication of ECMmimetic hydrogels, the two-photon-induced polymerization (2PP) strategy presents the most promise because it enables the 3D fabrication of user-dictated shapes with submicronscale resolution. ${ }^{8,9}$ High spatial resolution is accessible because the two-photon excited events are localized in a small volume within the focus of the laser beam (Scheme 1). By scanning the focused laser beam within a photopolymerizable precursor solution, well-defined hydrogel structures can be produced from a CAD model with true 3D control. In addition, in contrast to traditional stereolithographic techniques which require ultraviolet light, $2 \mathrm{PP}$ is induced by a near-infrared laser (typical wavelength: $800 \mathrm{~nm}$ ). This light source is far less harmful to surrounding tissues and also allows higher penetration depth, ${ }^{10}$ which makes it attractive for potential clinically relevant applications.

Several researchers have utilized two-photon technology to spatially functionalize preformed hydrogel matrices, by either immobilization or photorelease of bioactive ligands. ${ }^{11-14}$ For example, Hahn et al. reported the creation of 3D patterns and gradients of a cell-adhesive RGDS peptide

Additional Supporting Information may be found in the online version of this article.

(C) 2013 Wiley Periodicals, Inc.

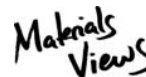




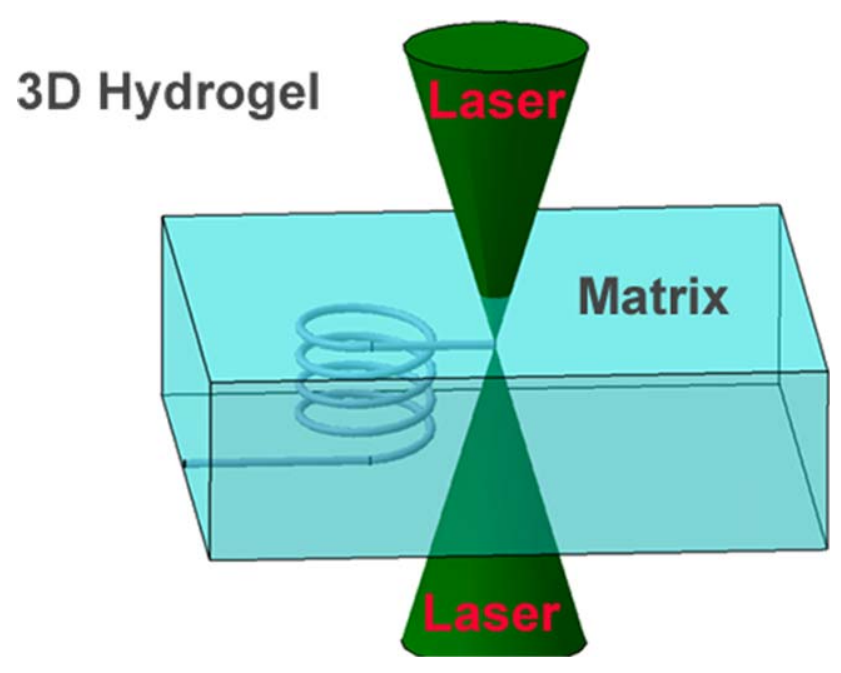

SCHEME 1 Schematic illustration of three-dimensional microfabrication of hydrogels via 2PP technique. [Color figure can be viewed in the online issue, which is available at wileyonlinelibrary.com.]

within polyethyleneglycol diacrylate (PEGDA)-based hydrogels. ${ }^{11}$ Cell migration activities were precisely controlled in the region patterned with RGDS. Nonetheless, up till now, very few groups have employed the $2 \mathrm{PP}$ technique to directly create 3D hydrogel scaffolds with complicated geometries. ${ }^{15-17}$

Seminal work of Campagnola et al. ${ }^{18}$ have proved that delicate gel microstructures could be fabricated out of native BSA by using a two-photon active photosensitizer. However, it is important to note that such crosslinking reactions are based on a di-tyrosine crosslinking mechanism. ${ }^{19}$ From a chemical point of view, the efficiency of di-tyrosine crosslinking is quite limited when compared to radical-mediated polymerization (chain-growth or step-growth) where hundreds of molecules are crosslinked via one initiating radial. In the former process, two separate tyrosyl radicals must form and then find one another to form only one crosslink. By comparison, radicalmediated polymerization seems to be a more efficient strategy for direct assembly of 3D hydrogels via 2PP.

While commercially available PEGDA has been widely used as hydrogel precursor due to its high reactivity and tunable physical properties, its biocompatibility is still controversial for clinical applications. ${ }^{20,21}$ Unpolymerized PEGDA and unreacted acrylate groups can undergo Michael addition reactions with amino or thiol groups of proteins making these monomers potentially cytotoxic. Therefore, photopolymerizable monomers with low cytotoxicity are in demand for tissue engineering applications. We have previously demonstrated that monomers based on vinyl esters are intriguing alternatives of (meth)acrylates for bone tissue engineering. $^{22,23}$ In particular, cytotoxicity studies on osteoblasts proved that these monomers are one or two orders of magnitude less cytotoxic than their acrylates analogs. ${ }^{22}$ Although vinyl esters homopolymerize at rates much less than acrylates, copolymerization of vinyl esters with multi-

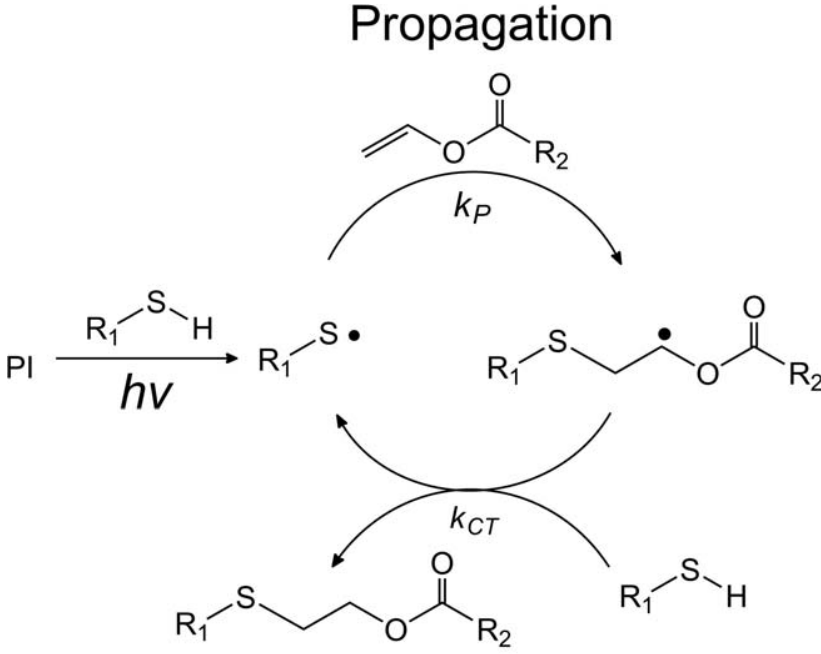

Highly efficient chain-transfer reactions

SCHEME 2 Proposed mechanism of radical-mediated thiolvinyl ester photopolymerization (PI, photoinitiator).

functional thiols proceeds at a sufficiently high rate to make the reaction suitable for most stereolithographic applications. ${ }^{24}$ The underlined mechanism of thiol-vinyl ester photopolymerization is proposed below (Scheme 2). Compared with acrylates, vinyl esters are less prone to chain-growth homopolymerization presumably because the formed radicals lack resonance stabilization. By contrast, reduced stability might also explain why these radicals show high reactivity toward chain transfer reactions with thiols. High reactivity and high conversion could be achieved in thiolvinyl ester photopolymerizations. ${ }^{24}$

One key advantage of photoinitiated polymerizations is their superior spatiotemporal control which is exploited by a variety of stereolithographic techniques. ${ }^{25-27}$ Seminal work of Anseth and coworkers have demonstrated that thiolnorbornene photopolymerizations are very promising models to create hydrogels with free-form patterns for in situ cell encapsulation and for noninvasive protein encapsulation studies. $^{28,29}$

Hydrogels prepared by thiol-vinyl ester photopolymerization remain unknown to our knowledge. With the intended application of generating artificial ECM hydrogel for tissue engineering, we chose to work with proteins that could be easily modified to provide thiol and vinyl ester macromers. The macromer approach lends itself well to biological applications since less thiol and ene groups need to react to form a gel and thus less heat is released.

Gelatin, a collagen-based cell-adhesive protein, has been widely used in the biomaterial community as hydrogel precursor due to superior biocompatibility, injectability, and enzymatic degradability. ${ }^{30}$ In addition, the RGD sequences on 
gelatin are capable of supporting cell adhesion, spreading, and proliferation. To functionalize gelatin with vinyl groups and render it photocrosslinkable, one of the established methods is based on amidation of lysine units with methacrylic anhydride, giving gelatin methacrylamide (Gel-MA). ${ }^{31-}$ ${ }^{33}$ Human endothelial cells were shown to selectively adhere and spread on photopatterned Gel-MA hydrogel microstructures and also within microfluidic channels. ${ }^{32,33}$ Although Gel-MA is well suited to one-photon fabrication methods, methacrylate-based monomers generally suffer from low reactivity in 2PP applications. Furthermore, it is generally accepted that the reactivity of methacrylates cannot be optimized by using thiol-ene strategy. ${ }^{34}$ To address the reactivity issue, an acrylamide derivative of gelatin (Gel-Ac) has been prepared by a multistep synthesis. ${ }^{35}$ Although high reactivity could be achieved, Gel-Ac strongly diminished the metabolic activity of MG63 cells, presumably due to Michael addition side reactions between amino groups of cell proteins with the acrylamide moiety of Gel-Ac. ${ }^{35}$ As such, we turned to the synthesis of a vinyl ester derivative of gelatin, which is expected to be cytocompatible and enzymatically biodegradable. Moreover, we envisage that the thiol-ene concept would enhance the reactivity of vinyl esters and thus enable the 2PP microfabrication of 3D hydrogels.

We herein report the synthesis and analysis of a vinyl ester derivative of gelatin hydrolysate (GH-VE) and investigate the use of GH-VE as hydrogel precursor for thiol-ene photopolymerization. Reduced bovine serum albumin (BSA-SH) has low cytotoxicity and has been previously investigated for use in drug delivery. ${ }^{36}$ It was thus used as model macrothiol where its multiple cysteine thiol groups efficiently react with the pendant vinyl groups on GH-VE. Finally, we explore the feasibility of microscale 3D photopatterning of combinations of these two proteins via two-photon-induced thiol-vinyl ester polymerization, providing hydrogel scaffolds with userdictated geometries.

\section{EXPERIMENTAL}

\section{Materials}

In this study, a commercially available enzymatic GH (SigmaAldrich, G0262) was used as starting material. It has a maximum molecular weight around 6000 Da. Dialysis tubing with a cutoff value of $3.5 \mathrm{~K}$ was purchased from Carl-Roth (Germany). Dialysis was always performed before use so that only selected gelatin fragments (MW: 3500-6000 Da) were used for derivatization. Anhydrous dimethylsulfoxide (DMSO) was freshly purified by vacuum distillation and kept dry with $4 \AA$ molecular sieves. The water content of DMSO was measured via Karl-Fisher moisture titration to be below 50 ppm. For photorheometry studies, Irgacure 2959 (I2959, BASF) was used as photoinitiator. For 2PP, a water-soluble photoinitiator (WSPI: 1,4-bis(4- $(N, N$-bis $(6-(N, N, N$-trimethylammonium)hexyl)amino)-styryl)-2,5-dimethoxybenzene tetraiodide) was synthesized according to literature. ${ }^{37}$ Bovine serum albumin (Fraction V) and divinyl adipate (DVA) were purchased from Sigma-Aldrich and TCI Europe, respectively.
Dulbecco's modified eagle medium (DMEM) was purchased from VWR Austria. The human osteosarcoma cell line MG63 was donated by LBI-Trauma (Vienna). All other chemical reagents or solvents were used without further purification.

\section{Characterization}

${ }^{1} \mathrm{H}$ NMR (400 MHz, $\mathrm{D}_{2} \mathrm{O}, 40{ }^{\circ} \mathrm{C}$ ) spectra were measured with a BRUKER AC-E 400 FT NMR-spectrometer. The chemical shift ( $\mathrm{s}=$ singlet, $\mathrm{bs}=$ broad singlet, $\mathrm{d}=$ doublet, $\mathrm{t}=$ triplet, $\mathrm{m}=$ multiplet) is given in ppm using the nondeuterated solvent as internal standard. Solvents with a grade of deuteration of at least $99.5 \%$ were used. GC-MS runs were performed on a Thermo Scientific DSQ II using a BGB 5 column ( $l=30 \mathrm{~m}, d=0.32 \mathrm{~mm}, 1.0 \mu \mathrm{m}$ film; achiral). UV-vis measurements were performed on a Perkin Elmer UV-vis Lambda 35 spectrophotometer.

MALDI-TOF Mass Spectrometry was performed on a Micromass TofSpec 2E time-of-flight mass spectrometer. The instrument is equipped with a nitrogen laser (337 nm wavelength, operated at a frequency of $5 \mathrm{~Hz}$ ), and a time lag focusing unit. Ions were generated by irradiation just above the threshold laser power. Positive ion spectra were recorded in reflectron mode applying an accelerating voltage of $20 \mathrm{kV}$ and externally calibrated with a suitable mixture of poly(ethyleneglycol)s (PEG). The spectra of 100-150 shots were averaged. Analysis of data was done with MassLynx-Software V4.1 (Micromass/Waters, Manchester, UK). Samples were dissolved in $1 \%$ TFA $\left(c=0.1\right.$ and $0.01 \mathrm{mg} \mathrm{mL}^{-1}$, respectively), 2,5-dihydroxybenzoic acid (2,5-DHB), and $\alpha$-cyano-4hydroxycinnamic acid (CHCA) were used as matrix $(c=10$ mg mL ${ }^{-1}$ in acetonitrile: $1 \%$ TFA $=70: 30(\mathrm{v} / \mathrm{v})$ ). Generally, solutions were mixed in the cap of a microtube in the ratio of $1 \mu \mathrm{L}: 10 \mu \mathrm{L}$. The resulting mixture $(0.5 \mu \mathrm{L})$ was spotted onto the target and allowed to air dry.

\section{Model Aminolysis Reaction}

To test the efficiency of the aminolysis reaction between $\varepsilon^{-}$ amino groups of lysine units and DVA, a model reaction with 1-hexyl-amine $\left(\mathrm{HexNH}_{2}\right)$ was performed. $\mathrm{HexNH}_{2}(0.1 \mathrm{mmol})$ was dissolved in anhydrous DMSO (5 mL) first. Then, this solution was added dropwise into an excessive amount of DVA (10 equiv., $1 \mathrm{mmol}$ ) at $50{ }^{\circ} \mathrm{C}$ under vigorous stirring. The reaction process was monitored hourly by GC-MS and ${ }^{1} \mathrm{H}$ NMR (see Supporting Information).

\section{Synthesis of a Vinyl Ester Derivative of Gelatin Hydrolysate}

Dialyzed GH (5.3 g, containing $2.01 \mathrm{mmol}$ lysine units) was dissolved in $100 \mathrm{~mL}$ anhydrous DMSO. Divinyl adipate (4.0 g, $20.1 \mathrm{mmol}$ ) was dissolved in $50 \mathrm{~mL}$ anhydrous DMSO and the GH/DMSO solution was added dropwise at $50{ }^{\circ} \mathrm{C}$ within $30 \mathrm{~min}$. After $24 \mathrm{~h}$ reaction at $50{ }^{\circ} \mathrm{C}$, the dark brown solution was distilled under high vacuum $\left(1 \times 10^{-2}\right.$ mbar, $45{ }^{\circ} \mathrm{C}$ ) to get rid of DMSO. Then the remaining slurry was dissolved in $300 \mathrm{~mL}$ distilled water and washed with ethyl acetate $(3 \times 100 \mathrm{~mL})$. The aqueous layer was dialyzed for $48 \mathrm{~h}$ with several changes of distilled water. After lyophilization, $3.5 \mathrm{~g}(61 \%)$ of GH-VE were obtained as orange solid. 


\section{Preparation of BSA Macrothiols}

BSA stock solutions (30 wt \%) were freshly prepared by dissolving the protein in phosphate buffered saline (PBS). Then varying amounts of the reducing agent tricarboxyethylphosphate (TCEP) were added to the BSA solution, to provide a 25 wt \% solution with varying concentration of free cysteines (see Supporting Information, Table S1). After $30 \mathrm{~min}$ incubation, the solution was dialyzed against PBS buffer under nitrogen protection for half a day with several changes of PBS. The sulfhydryl concentration was measured by Ellman's test. ${ }^{38}$ After calculation, the final concentration of free cysteines was checked to be $11.35 \mathrm{mM}$ (BSA-SH-3), 22.73 mM (BSA-SH-6), and $45.48 \mathrm{mM}$ (BSA-SH-12), respectively.

\section{Preparation and Characterization of Hydrogels 0-III}

Hydrogel precursor solutions consisted of GH-VE and BSA-SH along with 0.5 wt \% photoinitiator (either I2959 (one-photon) or WSPI (two-photon)) dissolved in PBS. Entry 0 contains 25 wt \% GH-VE with no BSA-SH. Entries I-III all contain 9 wt \% GH-VE and 16 wt \% BSA-SH where the latter has three different degrees of reduction such that $n_{\text {-SH }}: n_{\text {-ene }}$ $=0.1$ (I), 0.2 (II), and 0.4(III), (Supporting Information, Table S2). To prevent premature radical-mediated thiol-ene polymerization, $500 \mathrm{ppm}$ pyrogallol was added as stabilizer.

\section{Photorheology}

A plate-to-plate time-resolved photorheometer (Anton-Paar MCR-301) was used to monitor the one-photon curing kinetics of hydrogels during radical-mediated thiol-vinyl ester photopolymerization. Ultraviolet light (Omnicure S2000, 320-500 nm) was directed via a light guide though a glass plate to the underside of the sample. Light intensity at the cure surface was $10 \mathrm{~mW} \mathrm{~cm}{ }^{-2}$ as determined with an Ocean Optics USB $2000+$ spectrometer. After a 60 -s blank period, filtered UV light was triggered to irradiate the samples. Real-time measurements were made in oscillation mode, at $25{ }^{\circ} \mathrm{C}, 10 \%$ strain, $10 \mathrm{rad} \mathrm{s}^{-1}$, and $100 \mu \mathrm{m}$ thickness. Strain and frequency sweeps were always performed before and after polymerization to verify the linear response regime.

\section{Preparation of Hydrogel Pellets}

For cytotoxicity studies, hydrogel pellets were prepared in a multiwell PDMS chamber (well diameter: $6 \mathrm{~mm}$ ). Two hundred microliters of macromer solution was pipetted between two glass coverslips separated by a $1.5 \mathrm{~mm}$ spacer and then exposed to UV light $\left(20 \mathrm{~mW} \mathrm{~cm}^{-2}\right)$ for $300 \mathrm{~s}$. Pellets were detached from the slides and washed twice with sterile PBS. Finally, the pellets were incubated in DMEM so that macromer concentration was $1 \%$.

\section{Swelling Analysis}

For swelling analysis, hydrogel pellets were prepared as aforementioned and allowed to swell in PBS (pH 7.4) for 24 $\mathrm{h}$ at room temperature. The wet pellets were weighed to determine the equilibrium swollen mass $\left(M_{\mathrm{s}}\right)$ and then lyophilized to obtain the dry weight $\left(M_{\mathrm{d}}\right)$. The equilibrium mass swelling ratio was calculated as $M_{\mathrm{s}} / M_{\mathrm{d}}$. The experi- ments were repeated in triplicate to provide an average value with the standard deviation indicated by an error bar.

\section{MTT Assay}

The cytotoxicities of macromer solutions and hydrogel pellets were evaluated via MTT assay. The human osteosarcoma cell line MG63 was cultured in DMEM containing 10\% FBS. Macromer solutions of GH-VE and GH (1 and 0.1\%) were prepared in DMEM. MG63 cells were then seeded in a 96well tissue culture plate at a density of $1 \times 10^{4}$ cells per well in $200 \mu \mathrm{L}$ of DMEM containing $10 \%$ of fetal bovine serum. After $24 \mathrm{~h}$ incubation $\left(37{ }^{\circ} \mathrm{C}, 5 \% \mathrm{CO}_{2}\right)$, each of the macromer solutions and pellet extractions were added to the cells. After $24 \mathrm{~h}$ incubation, cells were washed twice with sterile PBS before the addition of $120 \mu \mathrm{L}$ of MTT reagents. Then the cells were incubated at $37{ }^{\circ} \mathrm{C}$ for $4 \mathrm{~h}$. The absorbance of the medium was measured at $540 \mathrm{~nm}$ using a microplate reader.

\section{Cell Adhesion}

For cell adhesion studies, hydrogel pellets were prepared in a similar approach as mentioned in the MTT assay. Pellets were transferred to a 24-well plate and covered with MG63 suspension $\left(5 \times 10^{4}\right.$ cells per well). After $24 \mathrm{~h}$ incubation $\left(37{ }^{\circ} \mathrm{C}, 5 \% \mathrm{CO}_{2}\right)$, supernatants were removed and samples were washed twice with PBS. Cells were stained using a LIVE/DEAD kit (Invitrogen). Fluorescence was visualized using an epifluorescence microscope (Leica DMI6000B).

\section{PP Microfabrication of Hydrogels}

Hydrogel precursor solutions were prepared as above with 0.5 wt $\%$ of the previously described WSPI. ${ }^{37} \mathrm{~A}$ Ti:sapphire laser system (HighQ Femtotrain $800 \mathrm{~nm}$, 80-fs pulse duration, $75-\mathrm{MHz}$ repetition rate) was used for $2 \mathrm{PP}$ microfabrication of hydrogels. Detailed information about the 2PP experimental setup and CAD-model design is available in the Supporting Information (Fig. S6).

\section{RESULTS AND DISCUSSION}

\section{Synthesis of GH-VE}

For the synthesis of GH-VE, the starting material was commercial GH derived from enzymatically degraded porcine collagen. After dialysis, $\mathrm{GH}$ has a molecular weight ranging from 3.5 to $6.0 \mathrm{KDa}$. Unlike other commercial sources of gelatin with higher molecular weight ( $>100 \mathrm{KDa}$ ), GH could achieve a maximum solubility at room temperature in PBS up to 50 wt \% without forming a physical gel, thus providing better processability.

To carry out the synthesis of GH-VE, an appropriate solvent had to first be determined. As DVA is prone to hydrolysis in the presence of water, the aprotic polar solvent DMSO was selected as the solvent since it avoids hydrolysis of DVA while providing good solubility for GH.

At the first stage of this work, a model reaction for the synthesis of GH-VE was performed on a low-molecular-weight model compound (1-hexyl-amine) in order to investigate the 


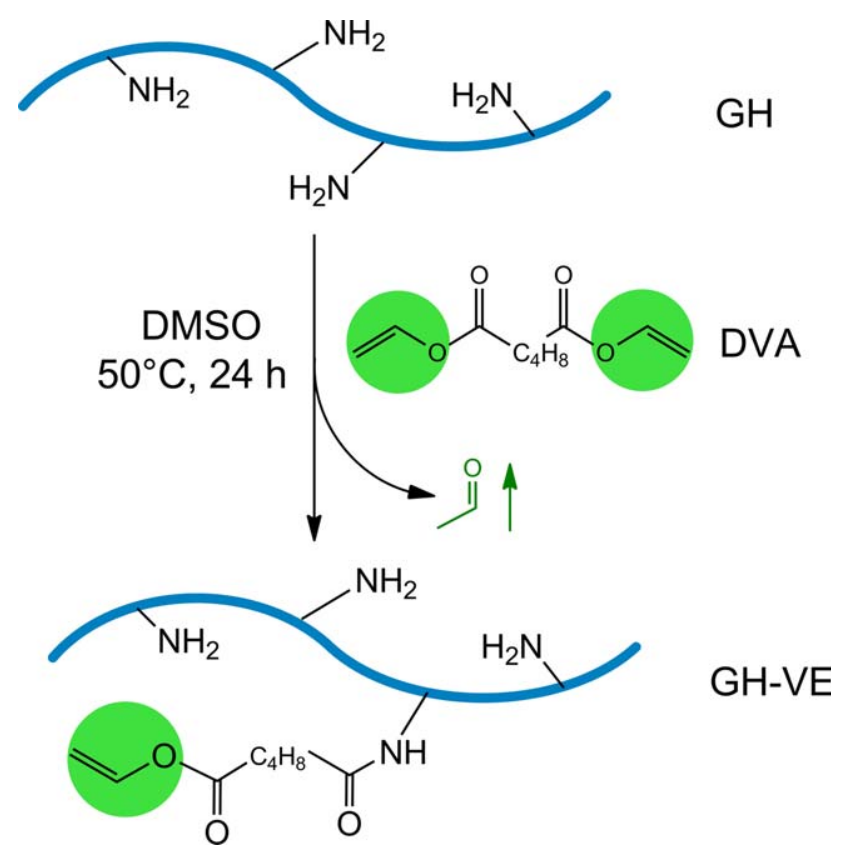

FIGURE 1 Synthesis of GH-VE. [Color figure can be viewed in the online issue, which is available at wileyonlinelibrary.com.]

efficiency of aminolysis reactions between $\varepsilon$-amino groups and a 10-fold excess of DVA (see Supporting Information Fig. $\mathrm{S} 1$ ). From the results of the model reaction (GC-MS analysis), 1-hexyl-amine was quantitatively converted (>98\%) with DVA after $3 \mathrm{~h}$ reaction in anhydrous DMSO at $50{ }^{\circ} \mathrm{C} .{ }^{1} \mathrm{H}$ NMR results provided further evidence on the successful modification. The proton signals at $2.66 \mathrm{ppm}$ which correspond to the methylene protons next to the amine groups disappeared entirely. Furthermore, only negligible amounts of diamides were detected in GC-MS experiments.
To modify GH with DVA moieties, one has to consider the reaction sites in $\mathrm{GH}$. Based on the model aminolysis reaction, DVA showed high reactivity towards primary amino groups. We assumed that there are two kinds of primary amino groups present in $\mathrm{GH}$ : first, the amino groups at the $\mathrm{N}$ terminus of $\mathrm{GH}$; second, the $\varepsilon$-amino groups on lysine units. The theoretical value of lysine units in GH was previously reported to be $0.38 \mathrm{mmol} \mathrm{g}^{-139}$ Based on an average molecular weight of dialyzed GH (4750 $\mathrm{g} \mathrm{mol}^{-1}$ ), around 1.8 lysine units on average should be present per GH peptide.

To functionalize these free amino groups with vinyl ester groups, we have treated GH with a 10-fold excess of DVA according to the conditions in the model reaction (Fig. 1). We hypothesized that the dropwise addition of GH solution into excessive DVA would minimize the possibility of intermolecular crosslinking by DVA. In order to remove the excessive DVA, DMSO was first removed by high vacuum distillation followed with a liquid - liquid extraction. After subsequent dialysis and lyophilization, the vinyl ester derivative of GH (GH-VE) was obtained as yellow powder. It is noteworthy that the only side product of this reaction is acetaldehyde (b.p., $20.2^{\circ} \mathrm{C}$ ) that can be easily removed.

As shown in Figure 2, the ${ }^{1} \mathrm{H}$ NMR spectrum of GH-VE was measured to characterize the product. Proton signal (a) at $7.36 \mathrm{ppm}$ corresponds to one of the unsaturated protons $\left(\mathrm{CH}_{2}=\mathrm{CH}-\mathrm{O}-\right)$ of the pendant vinyl ester group, while signals (b) and (c) from the other two unsaturated protons $\left(\mathrm{CH}_{2}=\mathrm{CH}-\mathrm{O}-\right)$ were partly overlapped with the solvent peak $\left(D_{2} O\right)$ within the range 5.2-4.7 ppm. Compared to the spectrum of GH (Supporting Information Fig. S2), an increase of the amide proton signal (d) could be observed at 3.55 ppm. Additionally, three kinds of methylene signals (f, g, e)

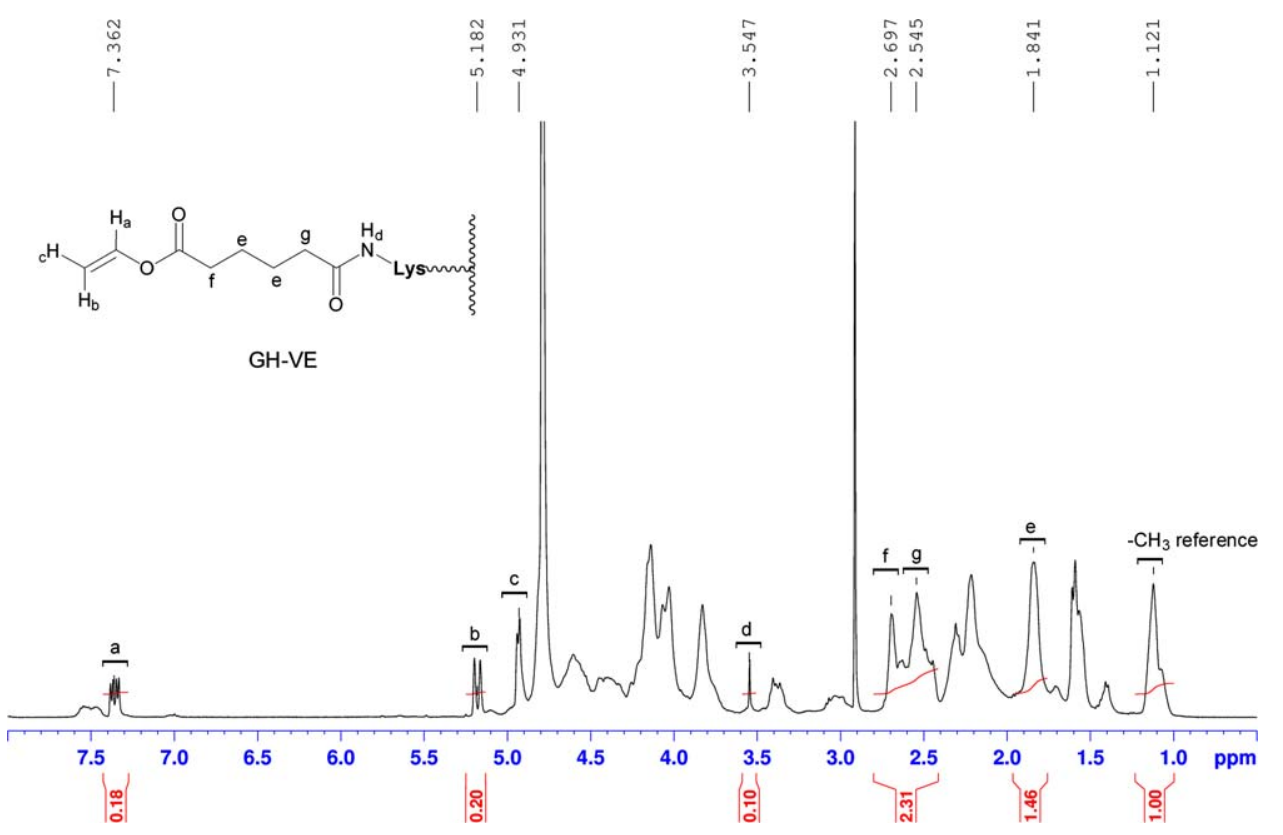

FIGURE $2{ }^{1} \mathrm{H}$ NMR spectrum of GH-VE $\left(\mathrm{D}_{2} \mathrm{O}, 40{ }^{\circ} \mathrm{C}, 400 \mathrm{MHz}\right)$. [Color figure can be viewed in the online issue, which is available at wileyonlinelibrary.com.] 


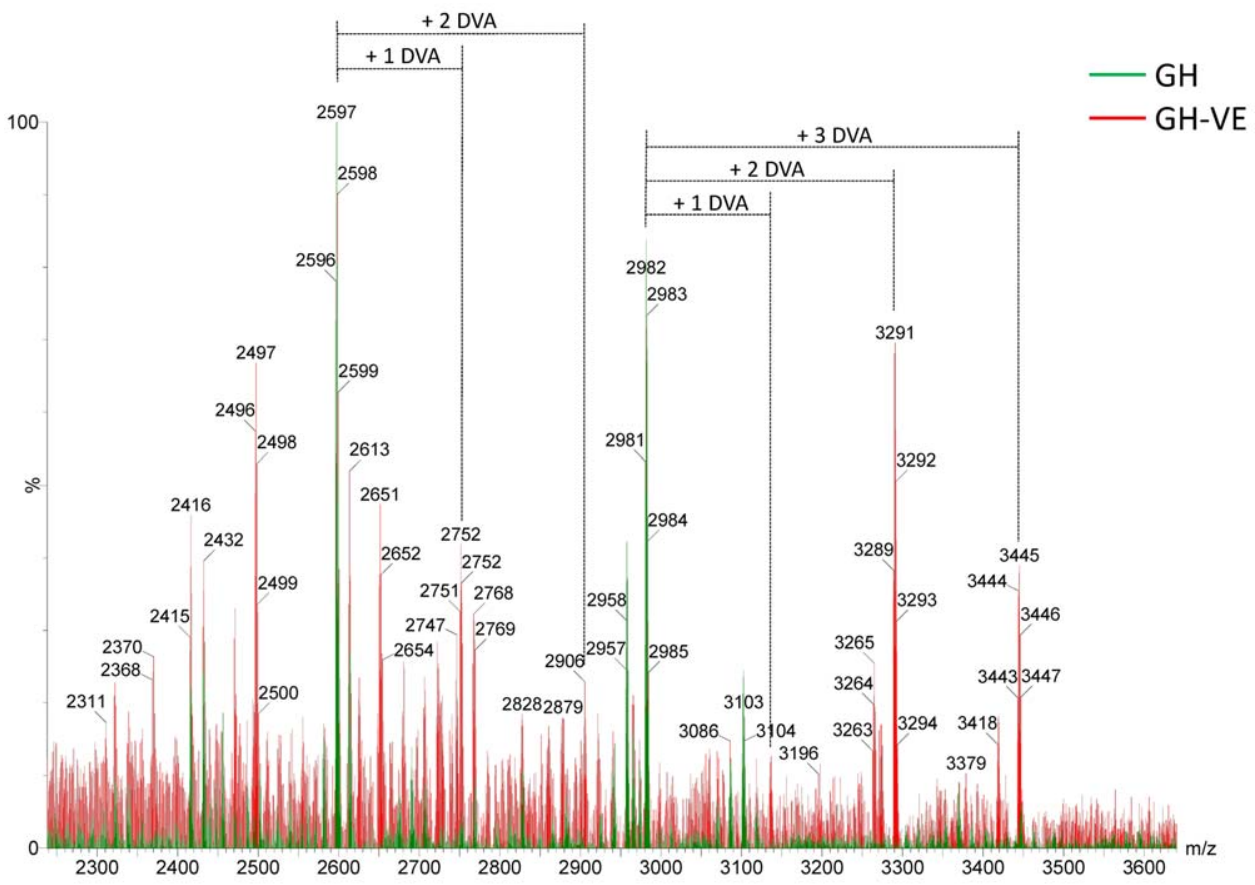

FIGURE 3 Overlaid MALDI-TOF mass spectra of GH (green) and GH-VE (red). Comparison of the fingerprints show that various signals observed for GH-VE can be correlated to peptides observed for $\mathrm{GH}$ assuming $n$-fold addition of divinyl adipate (DVA). [Color figure can be viewed in the online issue, which is available at wileyonlinelibrary.com.]

centered at 2.70-2.55 and 1.84 ppm showed obviously stronger signals due to the presence of DVA moieties. The combined evidence indicates that the aminolysis reaction of $\mathrm{GH}$ was successful.

To quantify the concentration of vinyl groups in GH-VE, the vinyl proton signal (a) at $7.36 \mathrm{ppm}\left(\mathrm{CH}_{2}=\mathrm{CH}-\mathrm{O}-\right)$ could be used as reference while the proton signal at 1.12 ppm corresponding to all $-\mathrm{CH}_{3}$ groups in $\mathrm{GH}$ could be used as internal standard. Gelatin from porcine skin has a content of $0.465 \mathrm{mmol} \mathrm{g}^{-1}-\mathrm{CH}_{3}$ groups originating from valine, leucine, and isoleucine residues. However, enzymatic degradation or a dialysis process would presumably change the $-\mathrm{CH}_{3}$ content. To roughly determine the final concentration of $-\mathrm{CH}_{3}$ groups in dialyzed $\mathrm{GH},{ }^{1} \mathrm{H} N M R$ spectrum with phenol as internal standard was measured (see Supporting Information Fig. S3). From this method, a concentration of $-\mathrm{CH}_{3}$ groups in $\mathrm{GH}$ was calculated to be $1.05 \mathrm{mmol} \mathrm{g}^{-1}$. Based on this value, the concentration of vinyl ester groups in GH-VE was calculated to be 0.57 mmol g ${ }^{-1}$ (see Supporting Information).

To design an efficient thiol-ene formulation for photocrosslinking, at least two thiol or ene functionalities should be incorporated to both thiol and ene macromers. ${ }^{25}$ To roughly estimate the number of DVA moieties per GH-VE, calculation resulted in about 2.7 DVA moieties on average were attached on the GH backbone (see Supporting Information). As this value was beyond the theoretical value of lysine units in $\mathrm{GH}$ (1.8 per $\mathrm{GH}$ ), we supposed that the amino group at the $\mathrm{N}$ - terminus contributed to this difference. Thus, the substitution degree of amino groups was calculated as $96 \%$.

Besides the NMR analysis, MALDI-TOF measurements were also performed to confirm the modification of GH. Spectra typical for complex mixtures of peptides were obtained for $\mathrm{GH}$ as well as for GH-VE. The spectra are overlaid in Figure 3 to facilitate direct comparison. The fingerprints of the spectra clearly indicate that divinyl adipate was bound to peptides between one and three times. For example, a rather intense peak in the spectrum of GH was observed at $m / z=$ 2981 Da (monoisotopic peak). After single modification with divinyl adipate, an increase of the mass of 154 Da should be observed due to introduction of the corresponding vinyl adipate moiety (Fig. 1). The corresponding signal at $3135 \mathrm{Da}$ was observed in the MALDI spectrum of GH-VE with low intensity. Signals with rather high intensities were also observed at 3289 and 3443 Da. These two signals are assigned to two-fold and three-fold modification of the peptide. As another example, the modification of a peptide with $2597 \mathrm{Da}$ is marked in Figure 3. Several other signals observed in the spectrum of GH-VE can also be correlated to signals of GH, for example, the signals at 3265/3419 Da can be interpreted as double/triple modification of the peptide originally observed at $2957 \mathrm{Da}$. Note, that these results correspond well to the average degree of modification of $\sim 2.7$, which was calculated from the NMR data.

Another important aspect during the synthesis of GH-VE was the possibility for intramolecular reactions of already 


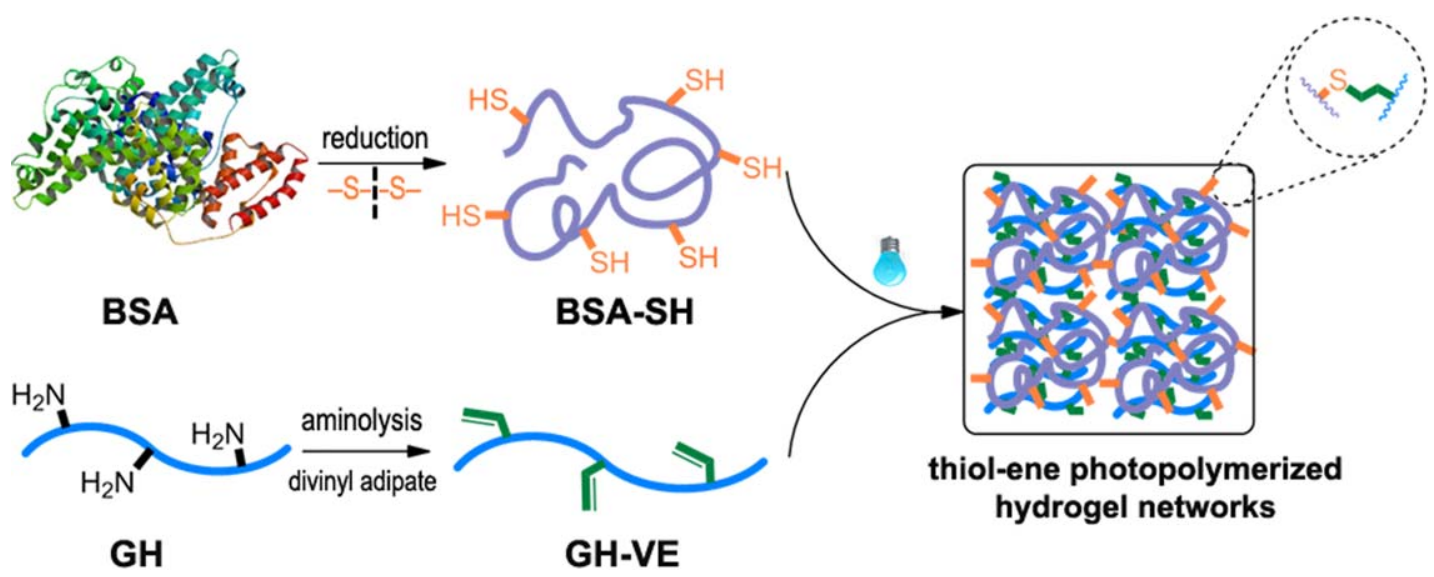

FIGURE 4 Hydrogel formation by thiol-ene photopolymerization using GH-VE and BSA-SH as precursors. [Color figure can be viewed in the online issue, which is available at wileyonlinelibrary.com.]

introduced vinyl ester groups with residual amino groups, an undesired side reaction that would lead to loss of polymerizable ene groups or potential gelation. The MALDI spectrum of GH-VE indicates that the side reaction was negligible. As one example, the signal at $m / z=3289$ Da was already assigned to a double-modified peptide. The species must contain at least one residual amino group, since threefold modification was observed too $(\mathrm{m} / \mathrm{z}=3443 \mathrm{Da})$. Consequently, an intramolecular side reaction would be possible. After loss of $\mathrm{C}_{2} \mathrm{H}_{4} \mathrm{O}$ (44 Da) the corresponding product should be observed at $3245 \mathrm{Da}$. As shown in Figure 3, this signal was not detected with reasonable intensity.

\section{Preparation of BSA Macrothiols (BSA-SH)}

The material selected to prepare water-soluble macrothiols was BSA, a 68-kDa protein widely used in biotechnology as tissue culture nutrient, blocking agent and enzymatic stabilizer. ${ }^{40}$ In addition to its wide availability and high solubility in aqueous media, BSA has a high number of cysteine residues making it a good candidate for thiol-ene chemistry. However, with the exception of a free thiol group at position 34 , most cysteines are locked in disulfide bridges. These bridges could fortunately be cleaved through simple reduction, providing multiple free cysteines which are photoclickable with vinyl groups of GH-VE in the presence of free radicals. As the rate of thiol-ene photopolymerization and the final mechanical properties of formed hydrogels depend on the thiol concentration, we hypothesized that by adding varying amounts of the reducing agent TCEP, the final concentration of free cysteines could be controlled and allow further control over the hydrogels' properties.

Although a series of early studies have shown that 17 disulfide bridges of BSA could be reduced completely and provide 35 free cysteines, the reported methods require very low BSA content $(<1$ wt $\%)$ due to the poor solubility of the denatured protein. ${ }^{40,41}$ For our purposes, protein concentration has to reach a certain level to maintain adequate mechanical properties of the resultant hydrogels. Practically, we found that the reduced BSA would precipitate out of PBS buffer if excessive TCEP was added. For instance, for a 30-wt $\%$ BSA solution, the maximum dose of reducing agent is around seven times the molar amount of BSA before precipitation was observed. By lowering BSA concentration, it was possible to achieve free cysteine/chain values from 2 up to 12. These reduced BSA macrothiols were however prone to reoxidize where stability was dependent on: (1) working concentration of BSA and (2) amount of TCEP added.

\section{Photopolymerization and In Situ Photorheology}

Since photoinduced reactions of unadultered native proteins (e.g., di-tyrosine crosslinking) lack efficiency, we selected to modify proteins with more reactive polymerizable moieties. Specifically, the radical-mediated thiol-ene click reaction was chosen as a polymerization strategy. Reduced BSA was used as a model macrothiol where its multiple cysteines can donate free thiol units to efficiently react with vinyl ester groups in modified gelatin (GH-VE, Fig. 4). BSA with different degrees of free cysteine units $(\sim 2-12$ cysteines per BSA molecule) were prepared and used while holding the weight ratio between GH-VE and reduced BSA constant. Because of a low number of thiols per weight, a high concentration of BSA (16 wt \%) was used. GH-VE was used at 9 wt \% to give a total macromer concentration of $25 \mathrm{wt} \%$. Reduced BSA with different degrees of free thiol was used in combination with GH-VE. By varying thiol to ene ratio $(0.1,0.2$, and 0.4$)$, a direct correlation of crosslink density to storage and loss modulus is provided.

To further characterize the photopolymerization process, in situ plate-to-plate photorheometry was applied. This method monitors the change in modulus during the liquid-to-solid transition in photocurable hydrogel precursors. ${ }^{42,43}$ The principle of photorheology is that the measured bulk modulus correlates with degree of polymerization and ultimately the crosslinking density. As the in situ rheometry technique is still limited to classical UV-curing, Irgacure 2959 was used as water-soluble photoinitiator due to its well-proved efficiency and cytocompatibility. ${ }^{44}$ Anseth and coworkers employed this method to monitor the time-resolved 


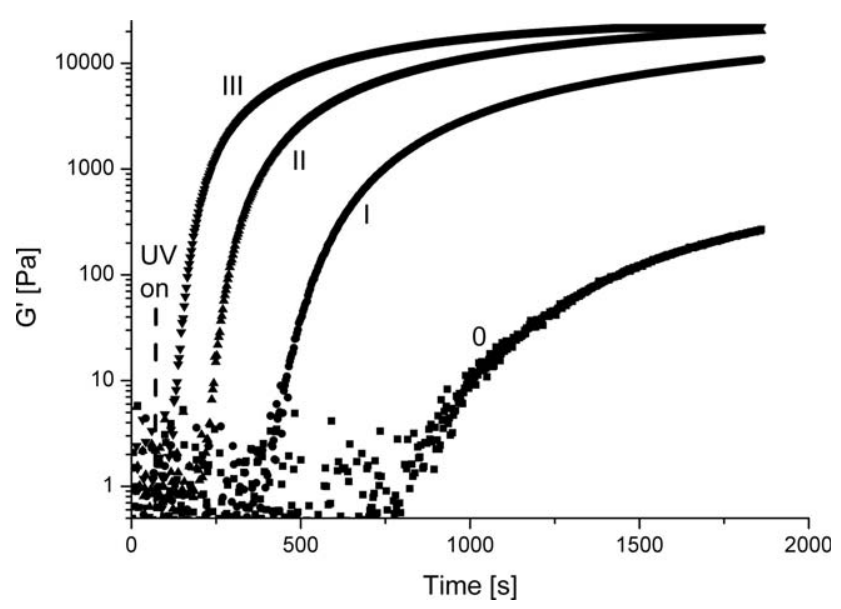

FIGURE 5 Plotted curve of time-resolved photorheometry. (entry 0: 25 wt \% GH-VE; entry I-III: 9 wt \% GH-VE, 16 wt \% BSA-SH-X $(X=3,6,12), n_{\text {thiol:ene }}=0(0), 0.1(\mathrm{I}), 0.2(\mathrm{II})$ and $0.4(\mathrm{III}) ; 0.5 \mathrm{wt} \% 2959$ in PBS; $100 \mu \mathrm{m}$ gap, $10 \mathrm{~Hz}, 10 \%$ strain, $10 \mathrm{~mW} \mathrm{~cm}^{-2}$ ).

evolution of elastic modulus during thiol-norbornene photopolymerization. Of particular relevance in tissue engineering applications, it was demonstrated that in situ rheometry was a promising method to study the enzymatic degradation process of PEG-peptide based hydrogels. ${ }^{28}$

While GH-VE was successfully synthesized, its photoreactivity was initially quite limited. Photorheology measurements (Fig. 5 , entry 0 ) showed that the homopolymerization of GH-VE took more than $1000 \mathrm{~s}$ to reach the gel point. As a result, reduced BSA was used as macrothiol to provide cysteines. Since it is accepted that cysteine-containing proteins are prone to oxidation due to disulfide recombination, we evaluated the stability of thiol-ene formulations (I, II, III) under varying conditions: (1) with initiator but without light (Supporting Information Fig. S4A); and (2) without initiator but with light (Supporting Information Fig. S4B). These control experiments indicated that the selected three formulations are stable under the selected conditions. To further investigate the influence of thiol/ene ratio on photoreactivity and final physical properties, formulations (0, I, II, III) were evaluated via in situ rheometry.

Both elastic modulus $\left(G^{\prime}\right)$ and loss modulus $\left(G^{\prime \prime}\right)$ of the samples could be monitored as a function of irradiation time. Gel points were determined in the vicinity of the $G^{\prime}$ and $G^{\prime \prime}$

TABLE 1 Influence of the Thiol/Ene Ratio on Gel Point and Elastic and Loss Modulus of Hydrogels 0, I, II, and III

\begin{tabular}{lllll}
\hline Entry & $n_{\text {-SH }}: n_{\text {-ene }}$ & Gel Point $(\mathrm{s})$ & $G^{\prime}(\mathrm{kPa})$ & $G^{\prime \prime}(\mathrm{kPa})$ \\
0 & 0 & 1120 & 0.27 & 0.07 \\
I & 0.1 & 442 & 10.87 & 1.15 \\
II & 0.2 & 205 & 20.24 & 1.84 \\
III & 0.4 & 95 & 22.11 & 2.01
\end{tabular}

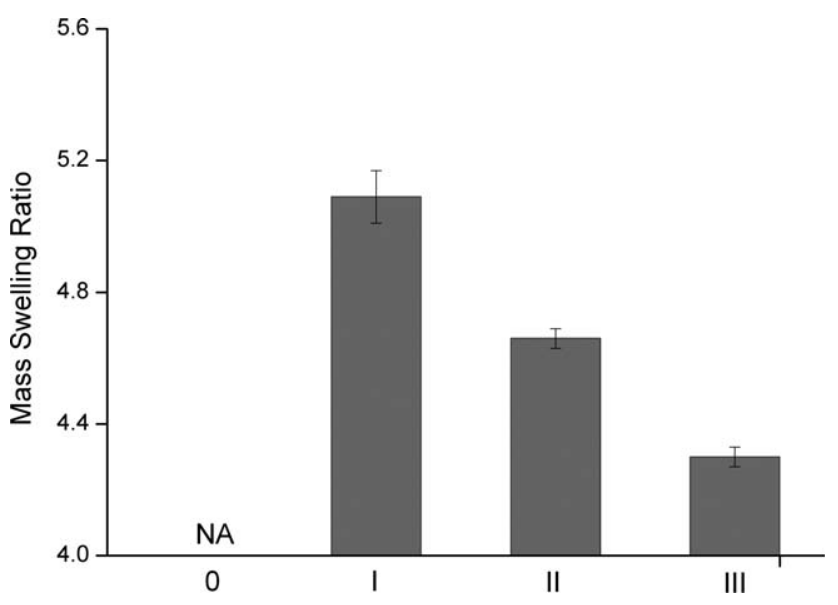

FIGURE 6 Mass swelling ratio of thiol-ene photopolymerized hydrogels with different thiol/ene ratio (either 25\% GH-VE (0), or $16 \%$ BSA-SH and $9 \% \mathrm{GH}-\mathrm{VE}, n_{\text {thiol:ene }}=0(0), 0.1(\mathrm{I}), 0.2(\mathrm{II})$ and $0.4(I I I))$. (NA: not applicable, error bars: $\pm S D$ ).

crossover (Supporting Information Fig. S4C). When the thiol/ ene ratio was increased from 0 to $0.1,0.2$, and 0.4 (Table 1), the gel points were shifted from 1120 to 442, 205, and $95 \mathrm{~s}$, suggesting increased reactivity. Meanwhile, elastic modulus increased from 0.27 to $10.9,20.2$, and $22.1 \mathrm{kPa}$, indicating an increased crosslinking density. Collectively, it was shown that the thiol/ene ratio could influence the onset of gelation and the crosslinking density of the network.

\section{Swelling Characteristics}

The swelling ability of a hydrogel is important for numerous applications since it influences both mechanical properties and solute transport activities. ${ }^{45}$ The extent to which a gel can swell is primarily dependent on the crosslinking density of the network. In this study, we evaluated the influence of thiol/ene ratio on swelling characteristics of GH-VE/BSA-SH hydrogels. In the case of the neat GH-VE hydrogel without BSA-SH (entry 0), the gel was too weak (Fig. 5) to accurately determine its swelling ratio. For gels containing BSA-SH (entries I-III), the extent of water uptake was found to depend on the concentration of thiol groups (Fig. 6). The mass swelling ratio decreased from 5.09 to 4.66 and 4.30 as the thiol/ene ratio increased from 0.1 to 0.2 and 0.4 , suggesting an increased crosslinking density of the network. These results are consistent with the photorheology measurements.

\section{Cytotoxicity}

Quantitative MTT assays were carried out to evaluate the cytotoxicities of macromer solutions [GH and GH-VE, Fig. 7(a)] and pellets extracts (I, II, III) on MG63 cells [Fig. 7(b)]. The results showed that GH-VE displayed negligible cytotoxicities after $24 \mathrm{~h}$ culture as compared to cell culture media (control). At the tested concentrations, cell viability was found to be even higher with GH-VE than with GH or the control. For pellets extracts, a similar phenomenon was also observed with extractions out of pellets (I, 
a) macromer solutions

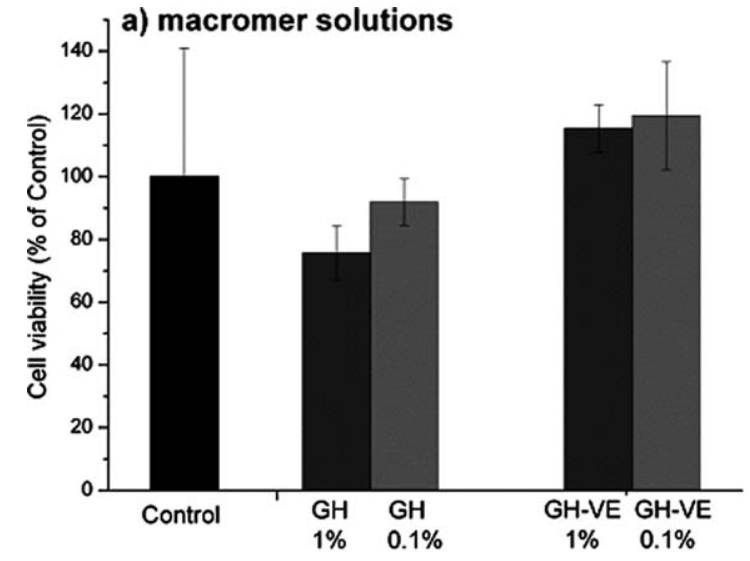

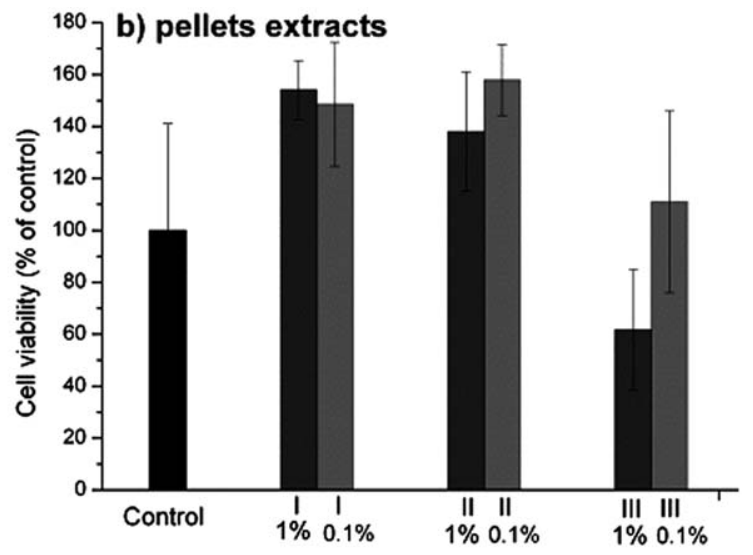

FIGURE 7 Metabolic activity of MG63 cells in contact with the macromer solutions (a: GH and GH-VE, 1 and $0.1 \%$, respectively) and pellet extracts (b: I, II, III) investigated by MTT assay (error bars: \pm SD, $n=3$ ).

II). In contrast, extractions out of pellets (III) decreased the cell viability to $70 \%$ of control. This might be due to the remaining traces of reducing agent while dialysis was performed before hydrogel preparation. These results were unexpected and the materials are undergoing further tests with different cell lines.

\section{Cell adhesion}

The ability for cells to adhere to a surface has important consequences on other functions including migration, proliferation, and apoptosis. Gelatin has a plurality of RGD motifs to promote cell adhesion. By contrast, BSA tends to resist cell adhesion due to its negative surface charge. ${ }^{46}$ We hypothesize that cell attachment could be adjusted by tuning the relative ratio between GH-VE and reduced BSA. As shown in Figure 8(a), when the relative ratio was 9:16, cells maintained round shapes, suggesting the pellets were not adhesive. Interestingly, when the ratio was increased to 12.5:12:5 [Fig. 8(b)] and 20:5 [Fig. 8(c)], there was increasing number of cells exhibiting a typical MG63 morphology. All together, these results indicate that the GH-VE supported cell adhesion. As reduced BSA tends to discourage adhesion, further work is underway using alternative disulfide-rich proteins that are also cell adhesive.

\section{Selection of Water-Soluble Two-Photon Initiator}

To carry out 2PP of hydrogels, one has to select a twophoton initiator (TPI) with high efficiency and good water solubility. Although researchers have used certain watersoluble dyes (e.g., eosin-Y) as TPI to fabricate hydrogels, ${ }^{47,48}$ these dyes generally lack efficiency due to their low twophoton-absorption (TPA) cross section. For instance, it was reported that the TPA cross-sectional value of eosin-Y was below 10 GM (Goeppert-Mayer units), which necessitates high laser powers for patterning. ${ }^{47}$ It has been reported that the 4,4'-dialkylamino bis(styryl)benzene (R1) chromophore shows a comparatively large TPA cross section (318 GM). ${ }^{49}$ The oil-soluble R1 has been utilized as a highly efficient initiator for 2PP microfabrication of acrylates. The underlined mechanism was thought to be a bimolecular electron transfer process from the photoexcited chromophore to acrylate monomers. ${ }^{50}$ Considering the efficiency of the R1 chromophore, we were interested to use the water-soluble analog (WSPI, Supporting Information Fig. S5) which has a much larger TPA cross-sectional value (144 GM) than eosin- $Y^{37}$
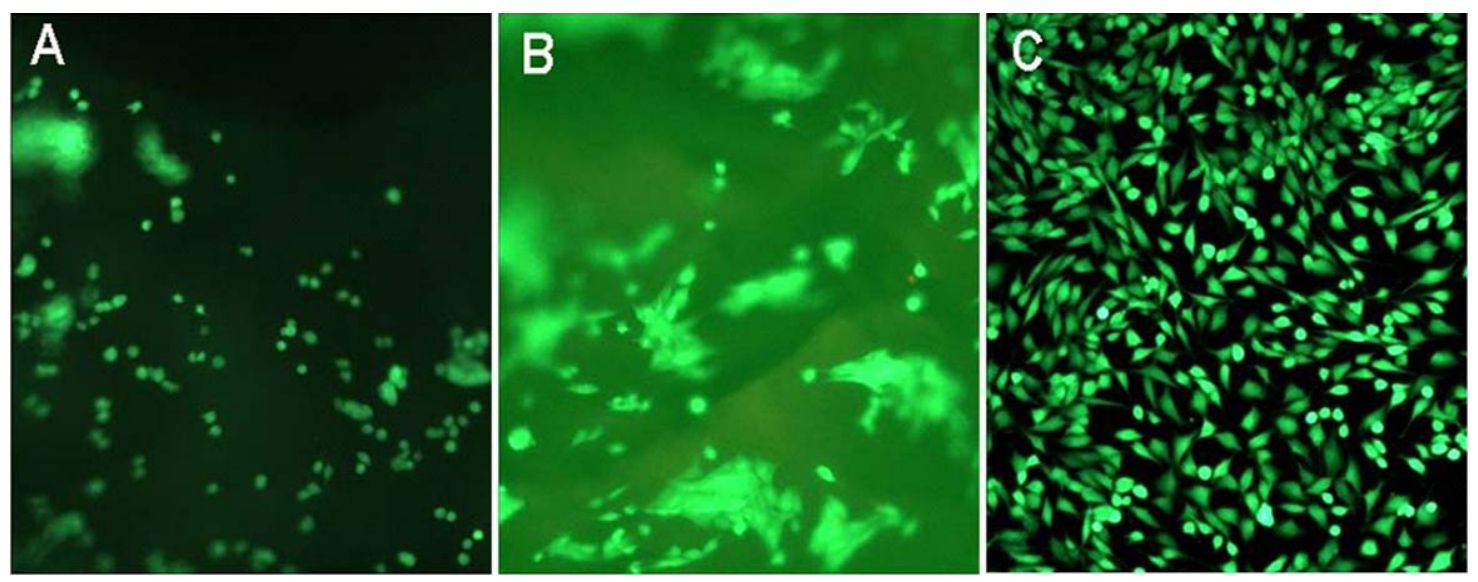

FIGURE 8 Cell adhesion could be regulated by changing the relative ratio between GH-VE and BSA-SH. (GH-VE \%: BSA-SH \% = 9:16 (a), 12.5:12.5 (b), and 20:5 (c); magnification: $100 \times$ ). [Color figure can be viewed in the online issue, which is available at wileyonlinelibrary.com.] 


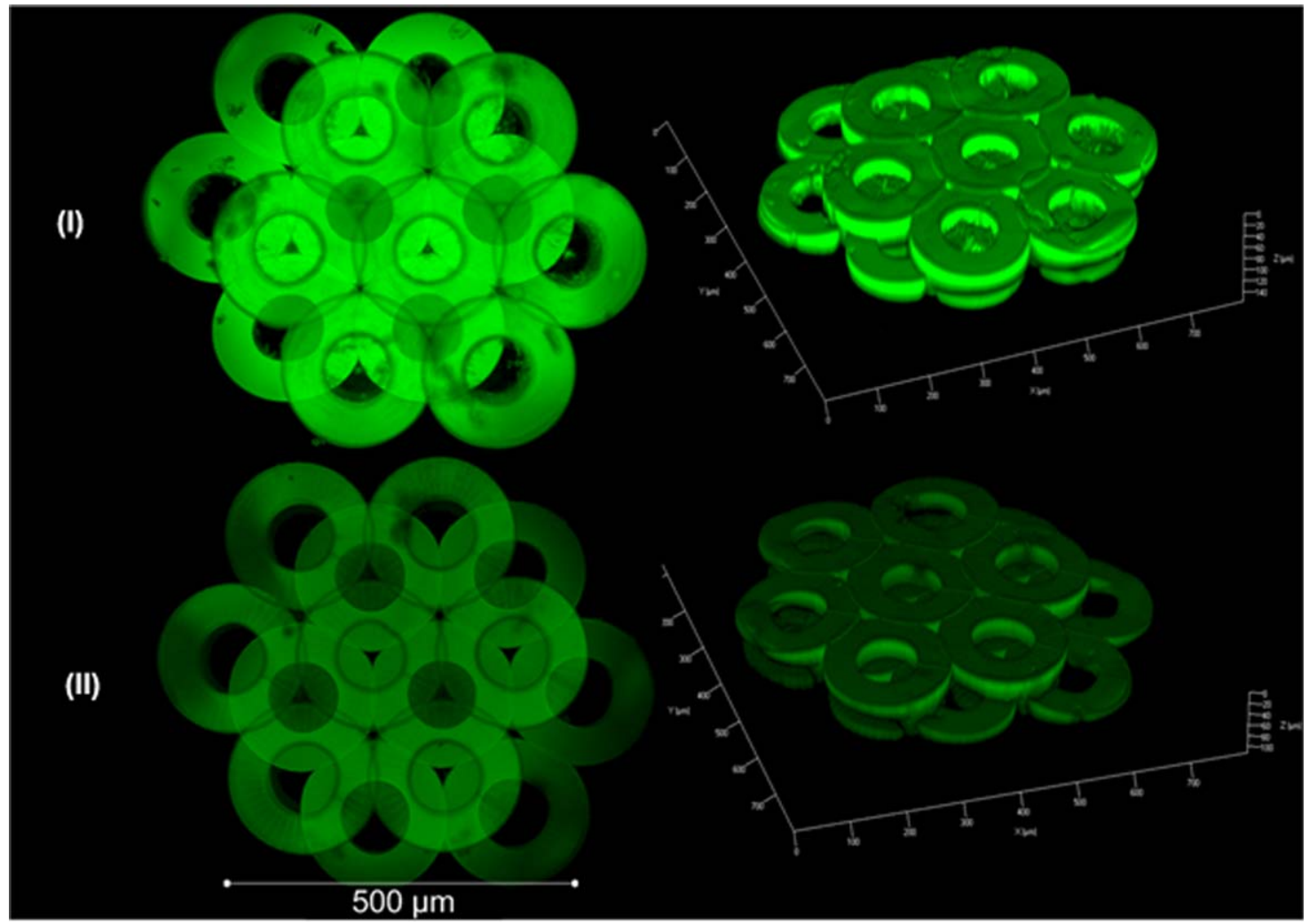

FIGURE 9 Two-dimensional and stacked three-dimensional LSM images of microfabricated hydrogel structures via 2PP. (16 wt \% BSA-SH-X $(X=3,6)$ and 9 wt $\%$ GH-VE, 0.5 wt \% WSPI in PBS, $n_{\text {thiol }}: n_{\text {ene }}=0.1$ (I) and 0.2 (II), respectively). [Color figure can be viewed in the online issue, which is available at wileyonlinelibrary.com.]

WSPI and its derivatives were previously studied to investigate the solvent effects in a variety of media for biological imaging applications. ${ }^{37}$ The addition of four quaternary ammonium pendant groups to the R1 chromophore provided enhanced solubility of WSPI in aqueous medium which is essential for biological applications. The charged end groups bonded to the aniline functionality through long aliphatic chains do not appear to interact with the electronic structure of the TPA chromophore and thus do not affect its photoinitiating behavior. To our knowledge, very few groups have used WSPI as photoinitiator for 2PP microfabrication of hydrogels. We previously explored the use of WSPI for the microfabrication of PEGDA-based hydrogels in the presence of C. Elegans. ${ }^{17,51}$ It was demonstrated that WSPI was a highly efficient water-soluble TPI which enabled a broad processing window and high writing speed $\left(10 \mathrm{~mm} \mathrm{~s}^{-1}\right)$.

\section{PP Microfabrication}

For tissue engineering applications, 3D hydrogel scaffolds with free-transport properties are critical to maintain cell functions since diffusive nutrient transport is only limited to short distances. ${ }^{6,52}$ Therefore, we designed a 3D CAD model (Supporting Information Fig. S7) consisting of three layers of packed cylinders with a hexagonal arrangement. We reason that the unique structural design would likely enable longterm culture of cells with high metabolic rate (e.g., hepatocytes). By using the 2PP technique, well-defined structures were written in accordance with the CAD model. Laser scanning microscope (LSM) images of the hydrogel constructs are shown in Figure 9. The structures' feature sizes were around tens of microns, which are relevant to the size range of most eukaryotic cells.

Generally, although high spatial resolution could be achieved by $2 \mathrm{PP}$, a major challenge still exists in terms of the long processing time which greatly limits potential applications. Very recently, we improved the current record of 2PP writing speed to a maximum level of $80 \mathrm{~mm} \mathrm{~s}^{-1}$ in nonaqueous media by using an optimized setup and an efficient TPI. ${ }^{53}$ However, ultrafast $2 \mathrm{PP}$ of hydrogels in the context of aqueous medium is more challenging when compared to $2 \mathrm{PP}$ in nonaqueous media, since hydrogel precursor solutions generally contain

TABLE 2 Influence of the Thiol-Ene Ratio on 2PP Fabrication Threshold

\begin{tabular}{lll}
\hline Entry & $n_{\text {-SH }}: n_{\text {-ene }}$ & Processing Window $(\mathrm{mW})$ \\
I & 0.1 & $75-375$ \\
II & 0.2 & $100-300$ \\
III & 0.4 & $50-125$
\end{tabular}

16 wt $\%$ BSA-SH-X $(X=3,6,12)$ and 9 wt \% GH-VE, 0.5 wt \% WSPI in PBS, $n_{\text {thiol }}: n_{\text {ene }}=0.1(\mathrm{I}), 0.2$ (II), and $0.4($ III), respectively. Writing speed: $15 \mathrm{~mm} \mathrm{~s}^{-1}$. 
much lower concentrations of initiators and monomers. Nevertheless, it is noteworthy that a comparatively high writing speed (50 $\mathrm{mm} \mathrm{s}^{-1}$ ) was achieved in this study compared to previously reported results (10 $\mathrm{mm} \mathrm{s}^{-1}$ for PEGDA) ${ }^{17}$ presumably due to the optimized fabrication setup and the robustness of two-photon-induced thiol-ene chemistry.

To investigate the influence of the thiol/ene ratio on the reactivity and the resultant $2 \mathrm{PP}$ processing window, comparative 2PP fabrication of simplified structures were performed with constant writing speed $\left(15 \mathrm{~mm} \mathrm{~s}^{-1}\right)$. It was found that all of the formulations were processable at $15 \mathrm{~mm} \mathrm{~s}^{-1}$. As shown in Table 2 , formulation-I $\left(n_{\text {thiol }}: n_{\text {ene }}=0.1\right)$ showed a broad processing window(75-375 mW) while formulation-II $\left(n_{\text {thiol }}: n_{\mathrm{ene}}\right.$ $=0.2$ ) showed a comparable processing window(100-300 $\mathrm{mW})$. Notably, formulation-III ( $n_{\text {thiol }}: n_{\text {ene }}=0.4$ ) could be processed in a power as low as $50 \mathrm{~mW}$, indicating that higher photoreactivity was obtained as a result of the increase of thiol concentration. However, the maximum processing power of formulation-III was much lower than formulation-I and formulation-II, which could be explained as the decrease of protein tolerance against light-induced denaturation when BSA was reduced to a certain extent.

\section{CONCLUSIONS}

As far as we know, direct assembly of customized 3D hydrogels via two-photon-excited thiol-ene chemistry is a new strategy in the field of tissue engineering. This work aims to introduce the thiol-ene concept into the field of 2PP microfabrication of hydrogels. Reduced BSA was applied as model macrothiols to donate varying amount of cysteines while GH-VE was successfully synthesized via an aminolysis reaction between GH and DVA. Importantly, GH-VE shows superior cytocompatibility and is the first reported vinyl ester derivative of a naturally derived macromolecule. However, from the synthetic point of view, it is important to note that the substitution degree of GH-VE is intrinsically limited to the lysine residue concentration in the starting material. As such, development of naturally derived macromolecules with a higher degree of vinyl ester groups is under way. We envisage that the fusion of rationally designed hydrogel precursors, the robust thiol-VE photoclick chemistry, and the 2PP technique would enable further biologically relevant studies to directly assemble 3D hydrogels with micrometer-scale resolution and user-defined characteristics (chemical composition, topography, ligand density, etc.). Such customized extracellular environments could lead researchers to a deeper understanding of how cells receive specific information from and interact with their ECM microenvironment.

\section{ACKNOWLEDGMENTS}

The authors acknowledge the China Scholarship Council for providing X-H. Qin a PhD fellowship (NO. 2009688014) and financial support by European Science Foundation (P2M network), PHOCAM project (NO. 260043) and ERC Starting Grant (NO. 307701) under the EU FP7. They also thank W.
Dazinger and C. Hametner for $400 \mathrm{MHz}$ NMR measurements and P. Gruber for assistance on 2PP fabrication.

\section{REFERENCES AND NOTES}

1 B. V. Slaughter, S. S. Khurshid, O. Z. Fisher, A. Khademhosseini, N. A. Peppas, Adv. Mater. 2009, 21, 33073329.

2 D. Seliktar, Science 2012, 336, 1124-1128.

3 M. W. Tibbitt, K. S. Anseth, Biotechnol. Bioeng. 2009, 103, 655-663.

4 C. A. DeForest, K. S. Anseth, Annu. Rev. Chem. Biomol. Eng. 2012, 3, 421-444.

5 L. G. Griffith, M. A. Swartz, Nat. Rev. Mol. Cell. Biol. 2006, 7, 211-224.

6 V. L. Tsang, S. N. Bhatia, Adv. Biochem. Eng. Biotechnol. 2007, 103, 189-205.

7 A. Atala, F. K. Kasper, A. G. Mikos, Sci. Transl. Med. 2012, 4, $160 \mathrm{rv} 112$

8 C. N. LaFratta, J. T. Fourkas, T. Baldacchini, R. A. Farrer, Angew. Chem. Int. Ed. Engl. 2007, 46, 6238-6258.

9 J. L. West, Nat. Mater. 2011, 10, 727-729.

10 P. A. Lapchak, Expert Rev. Med. Devices 2012, 9, 71-83.

11 M. S. Hahn, J. S. Miller, J. L. West, Adv. Mater. 2006, 18, 2679-2684.

12 A. M. Kloxin, A. M. Kasko, C. N. Salinas, K. S. Anseth, Science 2009, 324, 59-63.

13 Y. Luo, M. S. Shoichet, Nat. Mater. 2004, 3, 249-253.

14 M. W. Tibbitt, A. M. Kloxin, K. U. Dyamenahalli, K. S. Anseth, Soft Matter 2010, 6, 5100-5108.

15 S. J. Jhaveri, J. D. McMullen, R. Sijbesma, L. S. Tan, W. Zipfel, C. K. Ober, Chem. Mater. 2009, 21, 2003-2006.

16 A. Ovsianikov, M. Malinauskas, S. Schlie, B. Chichkov, S. Gittard, R. Narayan, M. Lobler, K. Sternberg, K. P. Schmitz, A. Haverich, Acta Biomater. 2011, 7, 967-974.

$17 \mathrm{~J}$. Torgersen, A. Ovsianikov, V. Mironov, N. Pucher, X. Qin, Z. Li, K. Cicha, T. Machacek, R. Liska, V. Jantsch, J. Stampfl, J. Biomed. Opt. 2012, 17, 105008.

18 P. J. Campagnola, J. D. Pitts, G. A. Epling, S. L. Goodman, Macromolecules 2000, 33, 1514-1523.

19 J. D. Spikes, H.-R. Shen, P. Kopečková, J. Kopeček, Photochem. Photobiol. 1999, 70, 130-137.

20 J. P. Van Miller, R. H. Garman, S. J. Hermansky, J. C. Mirsalis, C. B. Frederick, Regul. Toxicol. Pharmacol. 2003, 37, 54-65.

21 L. S. Andrews, J. J. Clary, J. Toxicol. Environ. Health 1986, 19, 149-164.

22 C. Heller, M. Schwentenwein, G. Russmueller, F. Varga, J. Stampfl, R. Liska, J. Polym. Sci. Part A: Polym. Chem. 2009, 47, 6941-6954.

23 B. Husár, C. Heller, M. Schwentenwein, A. Mautner, F. Varga, T. Koch, J. Stampfl, R. Liska, J. Polym. Sci. Part A: Polym. Chem. 2011, 49, 4927-4934.

24 A. Mautner, X. Qin, H. Wutzel, S. C. Ligon, B. Kapeller, D. Moser, G. Russmueller, J. Stampfl, R. Liska, J. Polym. Sci. Part A: Polym. Chem. 2013, 51, 203-212.

25 C. E. Hoyle, C. N. Bowman, Angew. Chem. Int. Ed. Engl. 2010, 49, 1540-1573. 
26 N. Gupta, B. F. Lin, L. M. Campos, M. D. Dimitriou, S. T. Hikita, N. D. Treat, M. V. Tirrell, D. O. Clegg, E. J. Kramer, C. J. Hawker, Nat. Chem. 2009, 2, 138-145.

27 M. J. Kade, D. J. Burke, C. J. Hawker, J. Polym. Sci. Part A: Polym. Chem. 2010, 48, 743-750.

28 B. D. Fairbanks, M. P. Schwartz, A. E. Halevi, C. R. Nuttelman, C. N. Bowman, K. S. Anseth, Adv. Mater. 2009, 21, 5005-5010.

29 J. D. McCall, K. S. Anseth, Biomacromolecules 2012, 13 2410-2417.

30 J. J. Rice, M. M. Martino, L. De Laporte, F. Tortelli, P. S. Briquez, J. A. Hubbell, Adv. Health Mater. 2013, 2, 57-71.

31 A. I. Van den Bulcke, B. Bogdanov, N. De Rooze, E. H. Schacht, M. Cornelissen, H. Berghmans, Biomacromolecules 2000, 1, 31-38.

32 J. W. Nichol, S. T. Koshy, H. Bae, C. M. Hwang, S. Yamanlar, A. Khademhosseini, Biomaterials 2010, 31, 55365544.

33 A. Khademhosseini, R. Langer, J. Borenstein, J. P. Vacanti, Proc. Natl. Acad. Sci. U S A 2006, 103, 2480-2487.

34 C. E. Hoyle, T. Y. Lee, T. Roper, J. Polym. Sci. Part A: Polym. Chem. 2004, 42, 5301-5338.

35 X.-H. Qin, Unpublished results.

36 A. Martínez, E. Muñiz, I. Iglesias, J. M. Teijón, M. D. Blanco, Int. J. Pharm. 2012, 436, 574-581.

37 H. Y. Woo, B. Liu, B. Kohler, D. Korystov, A. Mikhailovsky, G. C. Bazan, J. Am. Chem. Soc. 2005, 127, 14721-14729.

38 G. L. Ellman, Arch. Biochem. Biophys. 1959, 82, 70-77.

39 P. Koepff, K. Braumer, W. Babel, U.S. Patent 5733994, Deutsche Gelatine-Fabriken Stoess AG (DE), 1998. http://www.free patentsonline.com/5733994.html.

40 D. H. Tsai, F. W. Delrio, A. M. Keene, K. M. Tyner, R. I. Maccuspie, T. J. Cho, M. R. Zachariah, V. A. Hackley, Langmuir 2011, 27, 2464-2477.
41 Y. Wu, S. Chakrabortty, R. A. Gropeanu, J. Wilhelmi, Y. Xu, K. S. Er, S. L. Kuan, K. Koynov, Y. Chan, T. Weil, J. Am. Chem. Soc. 2010, 132, 5012-5014.

42 C. C. Lin, A. Raza, H. Shih, Biomaterials 2011, 32, 9685-9695.

43 C. A. Bonino, J. E. Samorezov, O. Jeon, E. Alsberg, S. A. Khan, Soft Matter 2011, 7, 11510-11517.

44 S. J. Bryant; C. R. Nuttelman; K. S. Anesth. J. Biomater. Sci. Polym. Ed. 2000, 11, 439-457.

45 J. L. Drury, D. J. Mooney, Biomaterials 2003, 24, 4337-4351.

46 M. C. Murphy, N. K. Howell, J. Sci. Food Agric. 1991, 55, 489-492.

47 P. J. Campagnola, D. M. Delguidice, G. A. Epling, K. D. Hoffacker, A. R. Howell, J. D. Pitts, S. L. Goodman, Macromolecules 2000, 33, 1511-1513.

48 J. C. Hoffmann, J. L. West, Integr. Biol. (Camb.) 2013, 5, 817-827.

49 M. Rumi, J. E. Ehrlich, A. A. Heikal, J. W. Perry, S. Barlow, Z. Hu, D. McCord-Maughon, T. C. Parker, H. Röckel, S. Thayumanavan, S. R. Marder, D. Beljonne, J.-L. Brédas, J. Am. Chem. Soc. 2000, 122, 9500-9510.

50 Y. M. Lu, F. Hasegawa, T. Goto, S. Ohkuma, S. Fukuhara, Y. Kawazu, K. Totani, T. Yamashita, T. Watanabe, J. Lumin. 2004, $110,1-10$.

51 J. Torgersen, X.-H. Qin, Z. Li, A. Ovsianikov, R. Liska, J. Stampfl, Adv. Funct. Mater. 2013, DOI: 10.1002/ adfm.201203880.

52 V. L. Tsang, A. A. Chen, L. M. Cho, K. D. Jadin, R. L. Sah, S. DeLong, J. L. West, S. N. Bhatia, FASEB J. 2007, 21, 790-801.

53 Z. Li, N. Pucher, K. Cicha, J. Torgersen, S. C. Ligon, A. Ajami, W. Husinsky, A. Rosspeintner, E. Vauthey, S. Naumov, T. Scherzer, J. Stampfl, R. Liska, Macromolecules 2013, 46, 352-361. 